\title{
Inhibition of miR-141 and miR-200a Increase DLC-1 and ZEB2 Expression, Enhance Migration and Invasion in Metastatic Serous Ovarian Cancer
}

\author{
Norhazlina Abdul Wahab ${ }^{1}$, Zahreena Othman ${ }^{1}$, Noor Wahidah Mohd Nasri ${ }^{1}$, \\ Mohd Helmy Mokhtar ${ }^{1}$, Siti Fatimah Ibrahim ${ }^{1}$, Adila A. Hamid ${ }^{1}$, Raja Affendi Raja Ali ${ }^{2}$ and \\ Norfilza Mohd Mokhtar ${ }^{1, *}$ \\ 1 Department of Physiology, Faculty of Medicine, Universiti Kebangsaan Malaysia, Kuala Lumpur 56000, \\ Malaysia; hazlina@ukm.edu.my (N.A.W.); othmanzahreena@yahoo.com (Z.O.) \\ 2 Department of Medicine, Faculty of Medicine, Universiti Kebangsaan Malaysia, Kuala \\ Lumpur 56000, Malaysia \\ * Correspondence: norfilza@ppukm.ukm.edu.my; Tel.: +60-3-9145-8610
}

Received: 6 March 2020; Accepted: 4 April 2020; Published: 17 April 2020

\begin{abstract}
The role of microRNA (miRNA) in ovarian cancer has been extensively studied as a regulator for its targeted genes. However, its specific role in metastatic serous ovarian cancer (SOC) is yet to be explored. This paper aims to investigate the differentially expressed miRNAs in metastatic SOC compared to normal. Locked nucleic acid PCR was performed to profile miRNA expression in 11 snap frozen metastatic SOC and 13 normal ovarian tissues. Functional analysis and regulation of their targeted genes were assessed in vitro. Forty-eight miRNAs were significantly differentially expressed in metastatic SOC as compared to normal. MiR-19a is a novel miRNA to be upregulated in metastatic SOC compared to normal. DLC1 is possibly regulated by miR-141 in SOC. MiR-141 inhibition led to significantly reduced cell viability. Cell migration and invasion were significantly increased following miRNA inhibition. This study showed the aberrantly expressed miRNAs in metastatic SOC and the roles of miRNAs in the regulation of their targeted genes and ovarian carcinogenesis.
\end{abstract}

Keywords: serous ovarian cancer; metastasis; microRNA; MIRN141 microRNA; MIRN200 microRNA

\section{Introduction}

An increasing trend of ovarian cancer was observed in countries with socioeconomic growth, which could be attributed to changes in lifestyle [1]. Ovarian cancer is divided based on its histological subtypes, which are serous, mucinous, endometrioid and clear cell types [2,3]. Serous ovarian cancer (SOC) stands as the most common histological subtype, which accounts for $70 \%$ to $80 \%$ of all ovarian cancer deaths [4]. Malignant serous tumors contribute to almost half of all malignant ovarian tumors, which occur most frequently in the sixth decade of life.

At the time of diagnosis, most malignant serous tumors would have spread extensively, mainly to the peritoneum, which reflects on the poor survival rates of the disease. The five-year survival rate for patients with stage III and stage IV SOCs are only $25 \%$ and $9 \%$, respectively [5]. Thus, the discovery of clinically useful biomarkers for diagnostic and therapeutic purposes is of utmost importance in overcoming the disease. A critical step towards achieving this goal is by establishing a unique repertoire of microRNA (miRNA) expression in metastatic SOC and normal ovarian tissues.

Over recent years, increasing evidence has revealed the roles of small non-coding single-stranded RNAs, called miRNAs, in ovarian cancer tumorigenesis and metastasis. Post-transcriptionally, miRNAs repress gene expression by recognizing complementary target sites in the $3^{\prime}$ untranslated region of targeted messenger RNA (mRNA). The expression of targeted mRNAs is silenced either by translational 
repression or by mRNA degradation. Each miRNA may target a number of different mRNAs and, similarly, a single mRNA can be targeted by several miRNAs [6,7]. Currently, it is well-known that aberrantly expressed miRNAs contribute to cancer development. High miRNA expression in a tumor may lead to oncogenesis via the downregulation of its targeted tumor suppressor, whereas low miRNA expression may cause oncogene activation [8]. MiRNAs involved in metastasis are denoted as metastamiRs, which play substantial roles in cell growth, epithelial-to-mesenchymal transition (EMT), cell adhesion, invasion and migration, apoptosis and angiogenesis [7]. In ovarian cancer, several reports have identified pro-metastatic and antimetastatic miRNAs. For instance, miR-205 has been shown to enhance motility [9], and miR-31 is believed to reduce cell migration and invasion in ovarian cancer cells [10].

Expression patterns of miRNAs correlate with ovarian cancer histological subtypes, tumor stage, chemoresistance, cancer recurrence and survival, making miRNAs valuable as tissue and blood-based biomarkers [11-14]. However, most of the miRNA profiling studies to date reported expression patterns across different ovarian cancer histological subtypes, specimen types (tumor tissues vs. cell lines), chemotherapy status (post-chemo vs. chemo naïve), heterogeneity of the tumor, tumor stage and grade, profiling platforms, RNA isolation methods and the type of normal controls used $[9,10,15,16]$. These differences contribute to the partial overlap of the differentially expressed miRNAs in ovarian cancer. In particular, very few profiling studies are looking specifically into the serous subtype of ovarian cancer with metastatic evidence. One study has successfully demonstrated how miRNA expression profiles differ in omental metastases of SOC compared to primary tumors and how these differences influence chemotherapy [17]. Another study identified exosomal miR-200b and miR-200c in the serum of patients with advanced epithelial ovarian cancer, suggesting the possible active role of exosomal secretion [18]. Our group has documented aberrantly expressed miRNAs in metastatic SOC compared to normal using formalin-fixed and paraffin-embedded tissue samples [11]. This study expands the body of current literature by examining miRNA expression profiles in metastatic SOC fresh tissue samples compared to normal and demonstrating how these miRNAs may influence ovarian cancer growth and progression.

\section{Materials and Methods}

\subsection{Human Tissue Samples}

Twenty-four unmatched snap frozen ovarian tissue samples were collected at the time of surgery at the Universiti Kebangsaan Malaysia Medical Centre (UKMMC) after obtaining informed consent from the patients. Ethics approval was obtained from the UKM Research Ethics Committee (Ref: UKM 1.5.3.5/244/UKM-GUP-2011-286). The tissue samples consisted of 11 SOC (stages III and IV) and 13 unmatched normal ovarian tissues. Cancer tissues were taken from primary sites with metastatic evidence in the peritoneal cavity, and normal tissues were taken from patients undergoing total abdominal hysterectomy with bilateral salphingo-oophorectomy for benign gynecological conditions. The consultant pathologist verified the cancer samples to be serous adenocarcinoma, with more than $80 \%$ of cancer cells present based on hematoxylin and eosin staining. The normal samples comprised of normal ovarian epithelial tissues and were confirmed to be free of any pathology, including benign cysts. All tissue samples were chemotherapy-naïve. The patient information of the ovarian tissue samples used in the study is shown in Table 1. 
Table 1. The patient information of the ovarian tissue samples used in the study.

\begin{tabular}{ccc}
\hline Characteristics & Cancer Samples (N (\%)) & Normal \\
\hline Number of samples & 11 & 13 \\
Mean age \pm S.D. (year) & $49.9 \pm 10.6$ & $51.9 \pm 6.7$ \\
\hline Race & & \\
Malay & $11(100 \%)$ & $5(38.5 \%)$ \\
Chinese & 0 & $6(46.2 \%)$ \\
Indian & 0 & $2(15.4 \%)$ \\
\hline Stage & & \\
III & $9(81.8 \%)$ & \\
IV & $2(18.2 \%)$ & \\
Low-grade & $1(9.1 \%)$ & \\
High-grade & $10(90.9 \%)$ & \\
\hline
\end{tabular}

\subsection{MicroRNA Expression Profiling and Validation}

Total RNA was purified using QIAzol lysis reagent and miRNeasy Mini Kit (Qiagen, Valencia, CA, USA; Cat No./ID: 217004) according to the manufacturer's instructions. RNA quantity and quality were determined using the RNA 6000 Nano Kit and Bioanalyzer 2100 (Agilent Technologies, CA, USA). RNA template, at a concentration of $5 \mathrm{ng} / \mu \mathrm{l}$, was reversed-transcribed to cDNA using Universal cDNA Synthesis Kit (Exiqon, Vedbaek, Denmark; Cat No: 203300). Differentially expressed miRNAs in cancer compared to normal was determined using miRCURY LNA Universal RT Cancer Focus microRNA PCR Panel, 96-well (V1.AF) and ExiLENT SYBR ${ }^{\circledR}$ Green Master Mix (Exiqon, Vedbaek, Denmark; Product No: 203820, 203823) according to the manufacturer's protocols. Eight upregulated miRNAs, namely miR-106a, miR-18a, miR-203, miR-93, miR-141, miR-7, miR-20a and miR-200a, were validated using the miRCURY LNA Universal RT Pick \& Mix PCR Panels (Exiqon, Vedbaek, Denmark; Product No: 203801).

\subsection{Statistical Analysis for microRNA Expression Profiling}

The $\mathrm{C}_{\mathrm{T}}$ values obtained from $\mathrm{qPCR}$ results were imported into the Exiqon GenEx Version 5.4.2 software (Exiqon, Vedbaek, Denmark) in .txt format. Data preprocessing was performed, and the reference gene was selected using NormFinder. MiRNA expression profiling was performed using the Kruskal-Wallis and LIMMA statistical tests with $p<0.05$ and fold-change between $<-2$ and $>2$. The data points that fell beyond the upper quartile +1.5 inter-quartile distance or the lower quartile -1.5 . inter-quartile distance in the box plot were considered outliers, and they were replaced with the group median. The lower and upper quartiles were defined by the 25th and 75th percentiles, respectively. The R programming software was then employed to generate the heatmap diagram and hierarchical clustering of the differentially expressed miRNAs in metastatic SOC compared to normal tissues.

\subsection{Bioinformatics Analysis of microRNA Targeted Genes}

The Oncomine database (http://www.oncomine.org) was employed to determine candidate genes involved in metastatic SOC. Predicted targeted genes for miR-141 and miR-200a were identified using TargetScan (http://www.targetscan.org), mirWalk (http://www.umm.uniheidelberg.de/apps/zmf/ mirwalk/mirnatargetpub.html) and miRDB (http://mirdb.org/miRDB). Gene lists from the Oncomine dataset and miRNA target prediction tools were overlapped using a Genn-Venn diagram to determine the overlapping candidate genes in all databases.

\subsection{Cell Culture and Transfection}

The ovarian cancer cell lines Caov3 (ovarian adenocarcinoma) and SKOV3 (ovarian adenocarcinoma, ascites) were obtained from the American Type Culture Collection (ATCC, Rockville, MD, USA) and cultured in DMEM and McCoy's 5A, respectively, supplemented with $10 \%$ fetal bovine serum and $1 \%$ penicillin-streptomycin. Transient transfections of all cells with miRCURY 
LNA anti-miR-141, anti-miR-200a and antisense control B (Exiqon, Vedbaek, Denmark; Product No: 4100001-4104908-001) were carried out using Lipofectamine 2000 (Invitrogen, Carlsbad, CA, USA). The miRNA inhibitors used were labeled with fluorescein (6-FAM) (Exiqon, Vedbaek, Denmark). The initial concentrations of both anti-miRs and control B were $50 \mu \mathrm{M}$. Cells were seeded in 24-well plates $24 \mathrm{~h}$ before transfection at a density of $40,000 \mathrm{cells} /$ well and cultured at $37^{\circ} \mathrm{C}$ in a $5 \% \mathrm{CO}_{2}$-humidified incubator. Transfection efficiency was assessed under the fluorescence microscope using phase contrast (PC) and fluorescein isothiocyanate (FITC) filter sets (Nikon, Tokyo, Japan).

\subsection{Relative Expression of DLC-1 and ZEB2}

We quantified the expression of DLC-1 and ZEB2 predicted to be targeted by miR-141 and miR-200a, respectively, using quantitative real-time polymerase chain reaction. Total RNA from the transfected cells were isolated at 24, 48 and $72 \mathrm{~h}$ using miRNeasy Mini Kit (Qiagen, Valencia, CA, USA). Up to $2 \mu \mathrm{g}$ of total RNA was reverse-transcribed to cDNA in a final reaction of $20 \mu l$ using the High-Capacity RNA to cDNA Kit (Applied Biosystem, Foster City, CA, USA; Cat No: 4387406) following the manufacturer's instructions. Gene expression was quantified using Taqman Pre-Designed Gene Expression Assays (Hs00183436_m1 for DLC-1 with NCBI reference: NM_001164271 and Hs002076091_m1 for ZEB2 with NCBI reference: NM_001171653.1) together with Taqman Fast Advanced Master Mix (Applied Biosystem, Foster City, CA, USA; Cat No: 4444964) in accordance to protocols. Relative expression was calculated using the comparative method with Hs02758991_g1 for GAPDH with NCBI reference: NM_001256799 as the calibrator. Experiments were done in duplicate. Statistical analysis was performed using a student's $t$-test, and $p<0.05$ was considered statistically significant.

\subsection{Cell Viability, Migration and Invasion Assays}

Cell viability, migration and invasion were assayed using PrestoBlue cell viability reagent (Thermo Fisher Scientific, Waltham, MA, USA), QCM 24-well Fluorometric Cell Migration Assay (Millipore, Billerica, MA, USA) and QCM 24-well Cell Invasion Assay (Millipore, Billerica, MA, USA), respectively. The assays were performed at 24,48 and $72 \mathrm{~h}$ post-transfection. Transfected cells stained with PrestoBlue reagent were incubated for $30 \mathrm{~min}$ at $37^{\circ} \mathrm{C}$ in $5 \% \mathrm{CO}_{2}$-humidified conditions. For migration and invasion assays, $300 \mu \mathrm{L}$ of cell suspension (containing $0.5-1.0 \times 10^{6}$ cells $/ \mathrm{mL}$ in chemo-attractant-free media) was added to the upper chambers (24-well inserts with 8 - $\mu$ m pore size). The lower chambers were filled with $500 \mu \mathrm{L}$ of media supplemented with $10 \%$ fetal bovine serum. Cells in the upper chambers were removed by pipetting out the remaining cell suspension after $24 \mathrm{~h}$ of incubation at $37^{\circ} \mathrm{C}$ in a $5 \% \mathrm{CO}_{2}$-humidified incubator. Migrated and invaded cells were dislodged completely from the underside of the inserts using the cell detachment solution and stained with lysis buffer/CyQuant GR dye solution. Fluorescence measurement for all the assays was performed on the Varioskan Flash ELISA reader (Thermo Fisher Scientific, Waltham, MA, USA). Functional assays were performed in duplicate. Statistical analysis was performed using a student's $t$-test, and $p<0.05$ was considered statistically significant.

\section{Results}

\subsection{Differentially Expressed microRNAs in Metastatic SOC Compared to Normal}

The NormFinder identified miR-27a with the lowest standard deviation, and this was chosen as the endogenous miRNA control to be normalized with all the samples. A total of 48 miRNAs were differentially expressed significantly in metastatic SOC compared to normal $(p<0.05)$, in which 22 and 26 miRNAs were up- and downregulated, respectively. The most significantly upregulated miRNAs with $\log _{2}$-fold changes $>2$ were miR-106a, miR-141, miR-182, miR-183, miR-18a, miR-19a, miR-200a/b/c, miR-205, miR-203, miR-20b, miR-21, miR-31 and miR-7. MiR-133a, miR-145, miR-195, miR-202, miR-1 and miR-100 were among the most downregulated miRNAs with $\log _{2}$-fold changes $<-2$. The complete list of significantly dysregulated miRNAs is presented in Table 2. 
Table 2. The list of differentially expressed miRNAs in metastatic serous ovarian cancer (SOC) compared to normal.

\begin{tabular}{|c|c|c|c|}
\hline miRNAs & $\begin{array}{c}\text { Kruskal-Wallis } \\
\text { (Adjusted } p \text { Value) }\end{array}$ & $\begin{array}{c}\text { Limma } \\
\text { (Adjusted } p \text { Value) }\end{array}$ & $\log _{2}$-Fold Change \\
\hline \multicolumn{4}{|l|}{ Upregulated } \\
\hline miR-141 & 0.000163 & $3.33 \times 10^{-14}$ & 9.419905 \\
\hline miR-200c & 0.000163 & $3.51 \times 10^{-11}$ & 8.867292 \\
\hline miR-200b & 0.000163 & $2.67 \times 10^{-11}$ & 7.872327 \\
\hline miR-205 & 0.000163 & $9.32 \times 10^{-11}$ & 7.804692 \\
\hline miR-200a & 0.000163 & $3.33 \times 10^{-14}$ & 7.694753 \\
\hline miR-182 & 0.000163 & $1.99 \times 10^{-12}$ & 6.125206 \\
\hline miR-183 & 0.000163 & $1.21 \times 10^{-10}$ & 4.882529 \\
\hline miR-7 & 0.000211 & $2.60 \times 10^{-6}$ & 3.953067 \\
\hline miR-203 & 0.000163 & $2.60 \times 10^{-6}$ & 3.227105 \\
\hline miR-18a & 0.000241 & $4.79 \times 10^{-7}$ & 3.065583 \\
\hline miR-31 & 0.027471 & 0.003668 & 2.604579 \\
\hline miR-20b & 0.000281 & $2.02 \times 10^{-5}$ & 2.461332 \\
\hline miR-21 & 0.000777 & $2.02 \times 10^{-5}$ & 2.115933 \\
\hline miR-19a & 0.007898 & 0.009027 & 2.069257 \\
\hline miR-106a & 0.000163 & $5.70 \times 10^{-10}$ & 2.032367 \\
\hline miR-20a & 0.000163 & $7.64 \times 10^{-8}$ & 1.914742 \\
\hline miR-93 & 0.000186 & $2.70 \times 10^{-6}$ & 1.849742 \\
\hline miR-17 & 0.003409 & $8.56 \times 10^{-5}$ & 1.668148 \\
\hline miR-106b & 0.000281 & $1.41 \times 10^{-5}$ & 1.337312 \\
\hline miR-210 & 0.013004 & 0.012497 & 1.202425 \\
\hline miR-30d & 0.005935 & 0.005966 & 1.202272 \\
\hline miR-15a & 0.001138 & 0.000167 & 1.197055 \\
\hline \multicolumn{4}{|c|}{ Downregulated } \\
\hline miR-202 & 0.000163 & $1.53 \times 10^{-8}$ & -5.74109 \\
\hline miR-133a & 0.000163 & $7.77 \times 10^{-9}$ & -4.25237 \\
\hline miR-145 & 0.000163 & $4.25 \times 10^{-8}$ & -4.24071 \\
\hline miR-195 & 0.000163 & $7.13 \times 10^{-7}$ & -4.04207 \\
\hline miR-125b & 0.000163 & $1.47 \times 10^{-11}$ & -3.82333 \\
\hline miR-100 & 0.000163 & $1.09 \times 10^{-10}$ & -3.67522 \\
\hline miR-1 & 0.000163 & $1.13 \times 10^{-7}$ & -3.47619 \\
\hline miR-143 & 0.000163 & $2.50 \times 10^{-7}$ & -3.41547 \\
\hline miR-214 & 0.000541 & $2.97 \times 10^{-5}$ & -3.39572 \\
\hline miR-99a & 0.000163 & $1.58 \times 10^{-10}$ & -2.79158 \\
\hline miR-9 & 0.000186 & $1.89 \times 10^{-8}$ & -2.73715 \\
\hline miR-150 & 0.000241 & $3.79 \times 10^{-6}$ & -2.55192 \\
\hline let-7b & 0.000241 & $2.04 \times 10^{-7}$ & -2.53452 \\
\hline miR-34a & 0.007898 & 0.000167 & -2.17351 \\
\hline miR-132 & 0.000777 & $7.67 \times 10^{-6}$ & -2.1695 \\
\hline miR-29a & 0.000211 & $7.96 \times 10^{-7}$ & -2.13643 \\
\hline miR-26a & 0.000777 & $1.03 \times 10^{-5}$ & -1.91756 \\
\hline let-7c & 0.000211 & $4.70 \times 10^{-7}$ & -1.84412 \\
\hline $\operatorname{miR}-10 \mathrm{~b}$ & 0.040545 & 0.00972 & -1.77229 \\
\hline $\operatorname{miR}-29 c$ & 0.002916 & 0.000189 & -1.69908 \\
\hline let-7a & 0.003409 & 0.000534 & -1.63707 \\
\hline miR-126 & 0.001138 & 0.000189 & -1.57362 \\
\hline miR-26b & 0.002517 & 0.000534 & -1.47826 \\
\hline $\operatorname{miR}-423-5 p$ & 0.000541 & $3.96 \times 10^{-5}$ & -1.41021 \\
\hline miR-148a & 0.007028 & 0.003807 & -1.25192 \\
\hline miR-29b & 0.017849 & 0.007969 & -1.07275 \\
\hline
\end{tabular}


The differentially expressed miRNAs in metastatic SOC compared to normal samples are represented by the principal component analysis (PCA) and the heatmap analysis, as shown in Figures $1 \mathrm{a}$ and $1 \mathrm{~b}$, respectively.

(a)

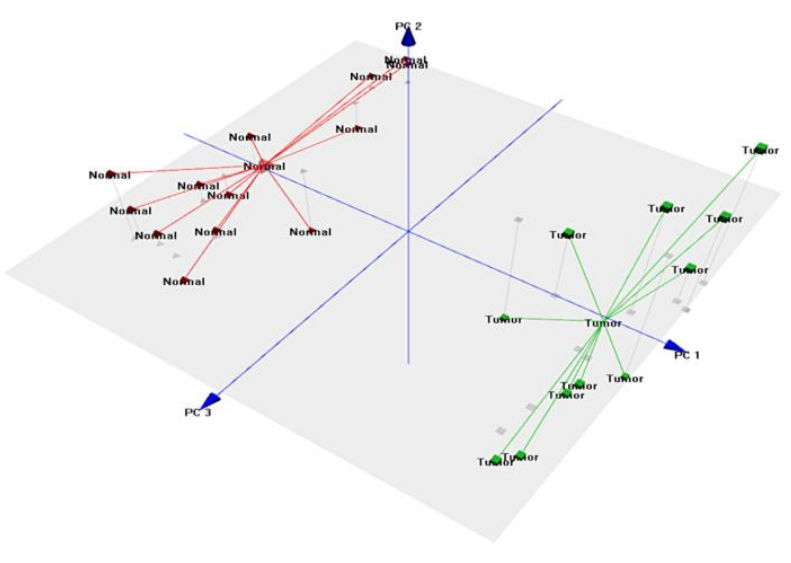

(b)

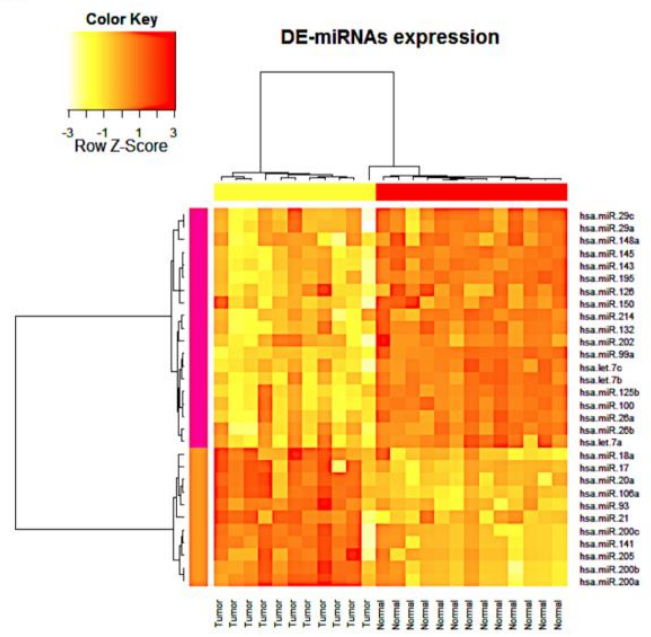

(c)

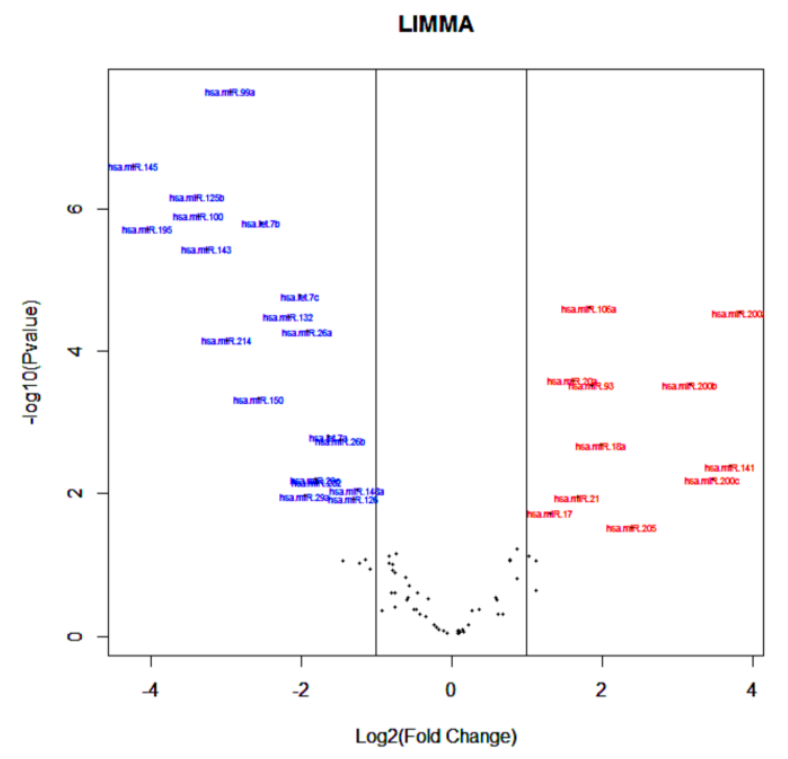

Figure 1. MicroRNA (MiRNA) expression profiling in metastatic serous ovarian cancer (SOC) compared to normal tissues. (a) Principal component analysis (PCA) shows the grouping of samples into cancer group (green lines) and normal (red lines). (b) Heatmap demonstrating the differentially expressed miRNAs in the tissue samples. Red shows upregulated miRNAs, and yellow shows downregulated miRNAs. (c) Volcano plot showing the relationship between the $-\log _{10} p$-value and the $\log _{2}$-fold change in metastatic SOC compared to normal.

The PCA and heatmap diagram clearly distinguished the samples into two different groups: metastatic SOC and normal samples. The volcano plot in Figure 1c shows the relationship between the $-\log _{10} p$-value and the $\log _{2}$-fold change in metastatic SOC compared to normal samples. Validation of the eight upregulated miRNAs confirmed the profiling results, as shown in Figure 2. 


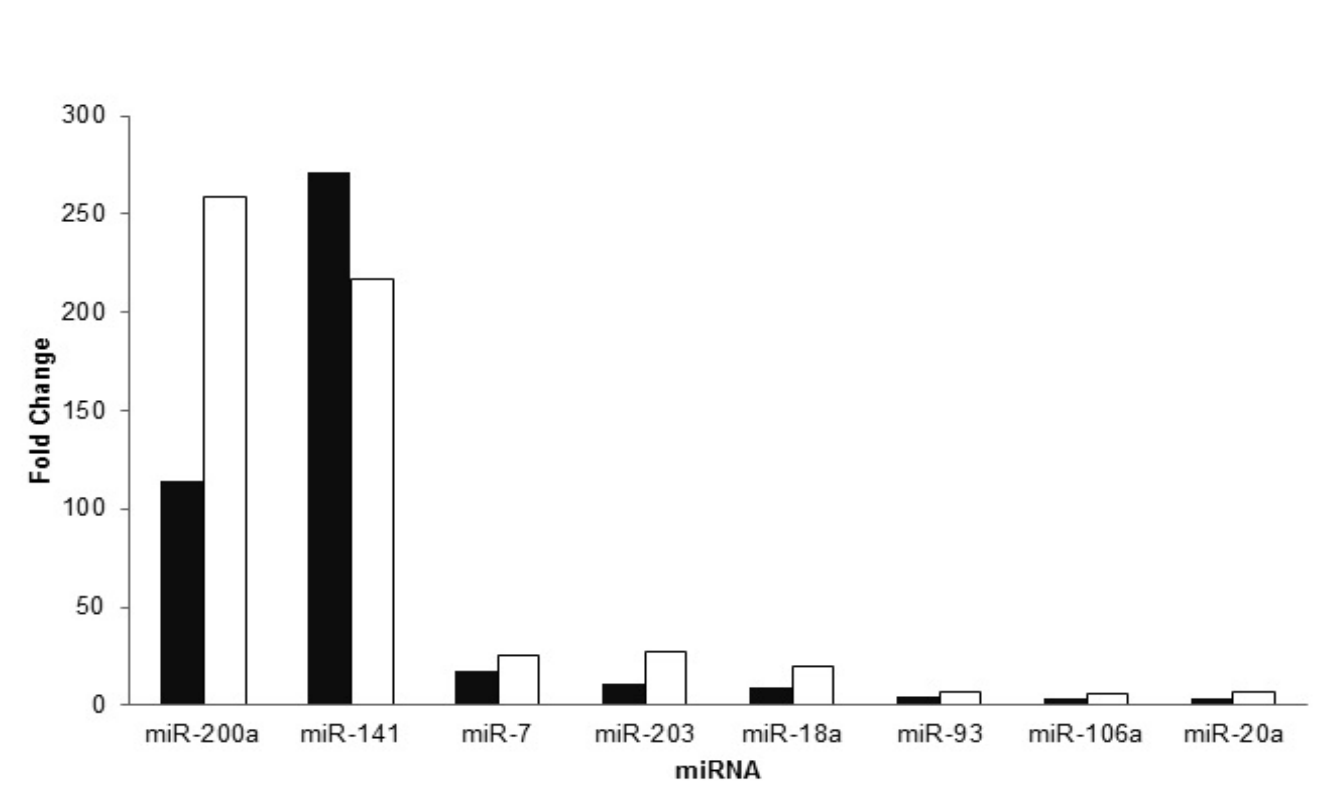

- Profiling

口Validation

Figure 2. Validation of eight upregulated miRNAs found in the profiling study. The validation data shows good concordance with the profiling data $(p<0.05)$, thus confirming the profiling results.

\subsection{Predicted Targeted Genes for miR-141 and miR-200a}

Oncomine search discovered the dataset from Adib et al. that listed a total of 1354 genes $(p<0.05)$ upregulated in SOC compared to normal. Analysis with TargetScan showed 744 predicted targeted genes for miR-141, 370 genes with miRWalk and 1100 genes with miRDB. MiR-200a was found to target 744 genes predicted by TargetScan, 357 genes by miRWalk and 1083 genes by miRDB. As shown in the Genn-Venn diagram (Figure 3), overlapping the datasets from the Oncomine and miRNA target prediction tools revealed ZEB2 and Deleted in Liver Cancer 1 (DLC-1) as the targeted genes for miR-141 and ZEB2 as the targeted gene for miR-200a.
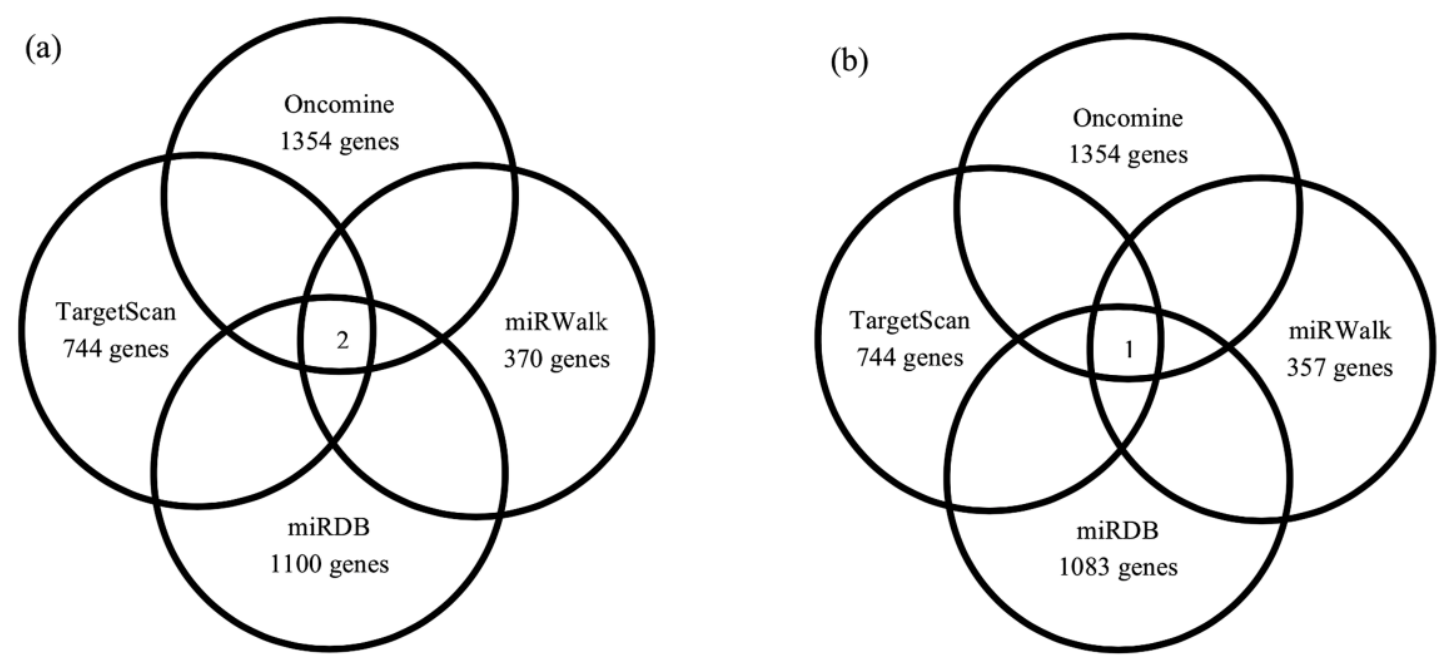

Figure 3. Venn diagrams showing the overlapping targeted genes for selected miRNAs as predicted by the bioinformatic analysis. (a) Two overlapping targeted genes for miR-141 were identified, namely DLC-1 and ZEB2. (b) MiR-200a is a single predicted miRNA to target the ZEB2 gene, which overlaps in all datasets. 


\subsection{The Expression of DLC-1 and ZEB2 Increased Following miR-141 and miR-200a Inhibition, Respectively}

Transfection efficiency was $60 \%$ to $70 \%$, as observed under the fluorescence microscope using a FITC filter set (Figure A1). As shown in Figure 4, the relative expression of DLC-1 and ZEB2 in Caov3 cells increased by 1.5- and 1.4-fold after $72 \mathrm{~h}$ of transfection with anti-miR-141 and anti-miR-200a, respectively. MiR-141 and miR-200a inhibition in SKOV3 cells caused the relative expression of DLC-1 and ZEB2 to rise by 1.2- and 1.5-fold, respectively, after $72 \mathrm{~h}$ of transfection (Figure 4). However, the changes observed were not statistically significant.
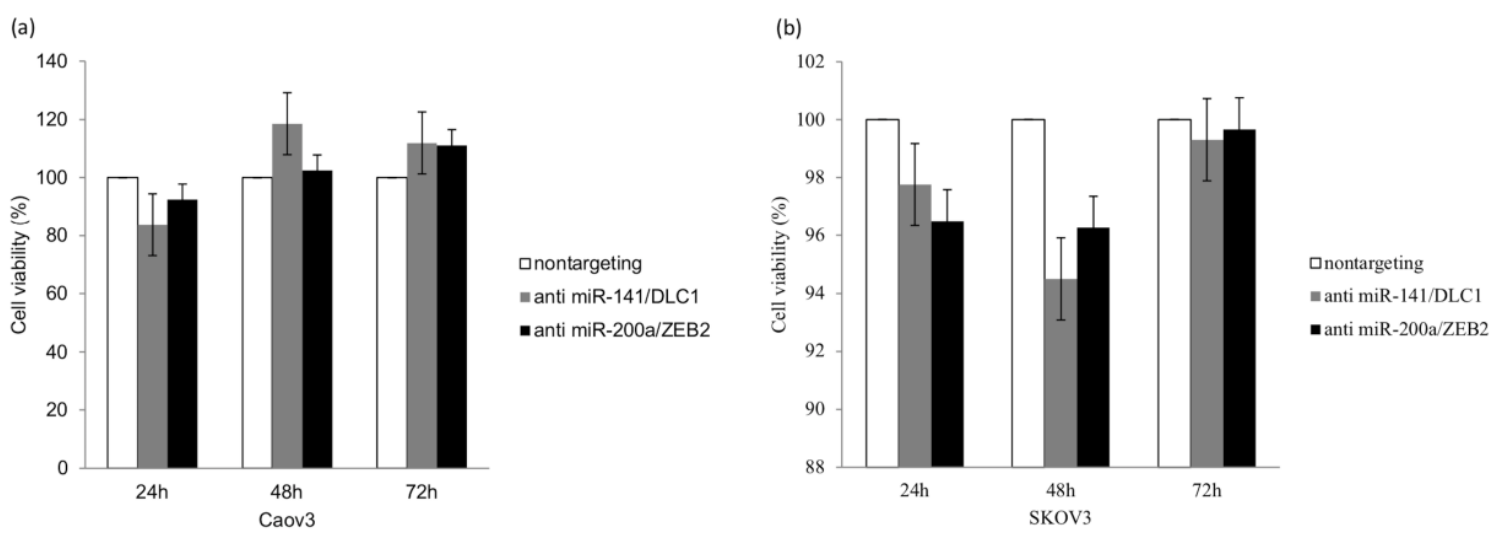

Figure 4. Relative expression of DLC-1 and ZEB2 increased following treatment with miRNA inhibitors. (a) In Caov3 cells, the relative expression of DLC-1 and ZEB2 increased after $72 \mathrm{~h}$ of transfection with anti-miR-141 and anti-miR-200a, respectively. (b) Similarly, miR-141 and miR-200a inhibition in SKOV3 cells led to increased DLC-1 and ZEB2 expression, respectively, after $72 \mathrm{~h}$. Statistical analysis was performed using a student's $t$-test. The graph represents the mean \pm standard error of duplicate experiments.

\subsection{MicroRNA Inhibition Reduced Cell Viability while Enhancing Cell Migration and Invasion}

As shown in Figure 5a, the percentage of viable Caov3 cells reduced significantly to $84 \%$ after $24 \mathrm{~h}$ of transfection with anti-miR-141 $(p<0.05)$. Significant reduction in cell viability $(94.5 \%)$ was also observed in SKOV3 cells after $48 \mathrm{~h}$ transfection with anti-miR-141 $(p<0.05)$. No significant decrease in cell viability was observed in both cells treated with anti-miR-200a, as shown in Figure 5a,b.

Transfection with anti-miR-141 in Caov3 cells increased the percentage of migrated cells significantly to $142 \%$ after $48 \mathrm{~h}$ (Figure 5c), while miR-200a inhibition caused the largest increase in cell migration (176\%) after $24 \mathrm{~h}(p<0.05)$, as shown in Figure 5d. However, miR-141 and miR-200a suppression did not appear to increase the percentage of migrated cells in SKOV3 cells, as shown in Figure 5 d.

As depicted in Figure 5e, inhibition of miR-141 and miR-200a led to a significant increase in the percentage of invaded cells in Caov3 and SKOV3 cells with the largest rise seen at $48 \mathrm{~h}(p<0.05)$. MiR-200a inhibition seemed to have the greatest effect on cell invasion, with a $621 \%$ and $735 \%$ upsurge of invading cells observed in Caov3 (Figure 5e) and SKOV3 cells (Figure 5f), respectively. 

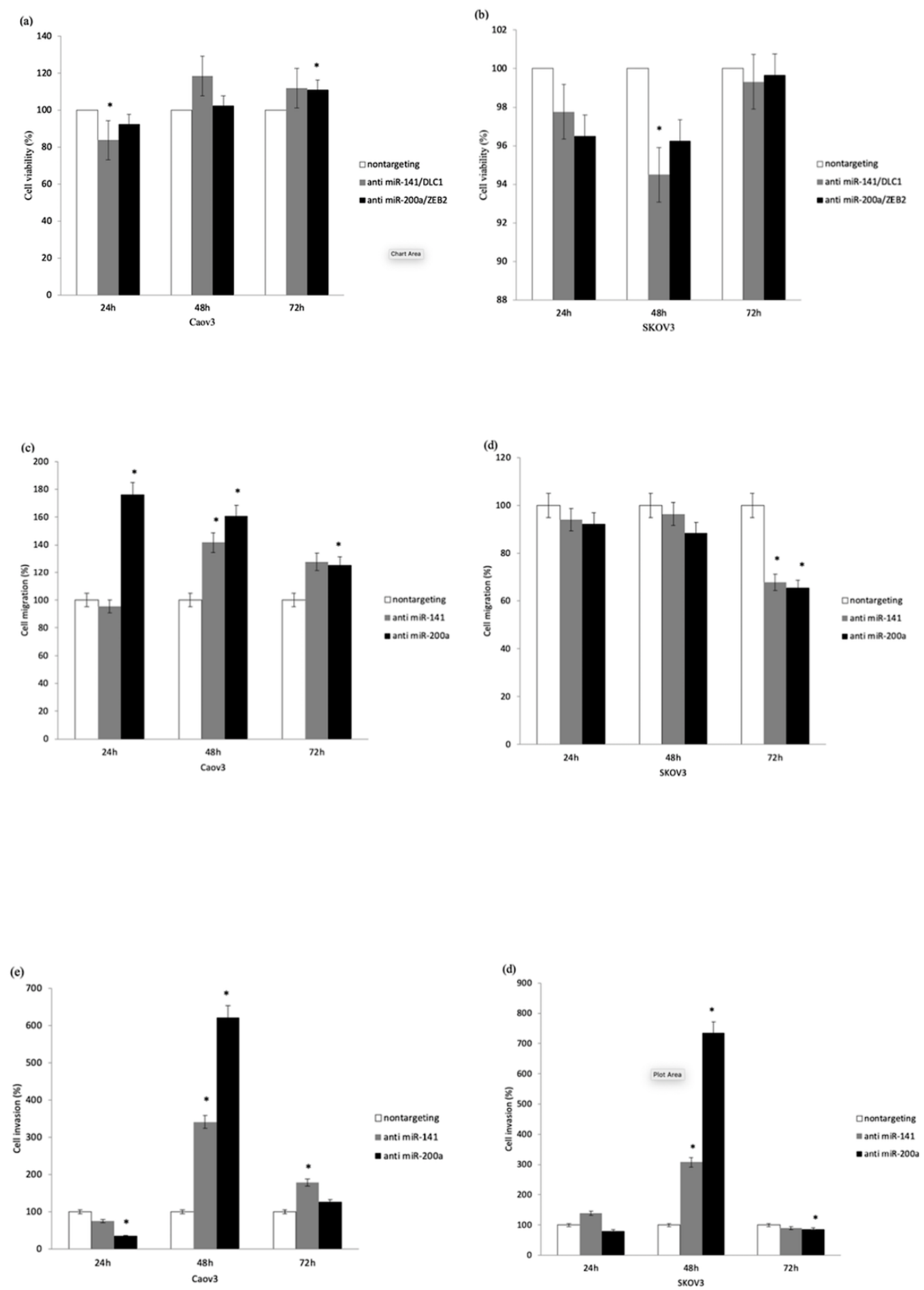

Figure 5. Functional assays performed on Caov3 and SKOV3 cells. (a) MiR-141 inhibition in Caov3 and (b) SKOV3 cells caused a significant reduction in cell viability after $24 \mathrm{~h}$ and $48 \mathrm{~h}$, respectively. Anti-miR-200a treatment did not cause a reduction in cell viability in both cell lines. (c) In Caov3 cells, treatment with anti-miR-141 caused enhanced cell migration significantly after $48 \mathrm{~h}$, while anti-miR-200a treatment led to the highest increase in cell migration after $24 \mathrm{~h}$. (d) Increased cell migration, however, was not observed in SKOV3 cells treated with miRNA inhibitors. (e,f) MiR-141 and miR-200a inhibition in both cell lines led to the highest significant increase in cell invasion after $48 \mathrm{~h}$. Statistical analysis was performed using a student's $t$-test. The graph represents the mean \pm standard error of duplicate experiments. ${ }^{*} p<0.05$. 


\section{Discussion}

This study was initiated with the hope of identifying histotype-specific miRNA expression patterns with a greater emphasis on cancers with metastatic potential. Many profiling studies have been done on ovarian cancer across different continents and populations, such as the United States of America, China, Korea and Taiwan [9,19-21]. In this paper, the differentially expressed miRNAs have been successfully categorized into two main clusters (up- and downregulated miRNAs) for the Malaysian population. Another Malaysian study has demonstrated the differentially expressed miRNAs in formalin-fixed paraffin-embedded (FFPE) ovarian tissue samples [10]. Our study complements the current literature by showing the aberrantly expressed miRNAs in fresh ovarian tissue samples.

A large number of the upregulated miRNAs determined from our profiling experiment were consistent with those which have been reported, namely miR-106a [19], miR-141 [10,19,22], miR-15a [20,22], miR-17 [19], miR-182 [10,16,19], miR-18a [10,22], miR-200a [10,19,22], miR-200b, miR-200c [10,19,22], miR-203 [10,22], mir-205 [10], miR-20a [22], miR-20b [10], miR-7 [10] and miR-93 [10]. However, the expression of miR-106b and miR-183 were inconsistent with the study by Dahiya et al. in which these miRNAs were found to be downregulated in epithelial ovarian cancer compared to normal [23]. MiR-21, miR-210 and miR-31 were found to have different expression in different studies [10,20,22]. The discrepancies in miRNA expression observed from these studies may be contributed by the different types of ovarian tissue samples used (fresh samples, FFPE and cells); histological subtypes of epithelial ovarian cancer included in the study (serous, endometrioid, mucin and clear cell); the profiling platforms used (qPCR, microarray and deep sequencing) and the normal tissues used as controls (whole normal ovary and normal ovarian surface epithelial cells).

Nevertheless, despite the many differences in the methods employed in different studies, good concordance of the overlapping upregulated miRNAs was observed, as shown in the list above. Potentially effective biomarkers for diagnostic, treatment or prognostic purposes may be unearthed from this unique overlapping miRNA list, thus providing useful insights for the ongoing translational research, from biomarker discoveries in the lab to clinical practices in the healthcare setting. This study is also the first to report on the upregulation of miR-19a in metastatic SOC compared to normal, making it a novel discovery. This new finding will certainly add to the existing knowledge of the upregulated miRNAs in metastatic SOC compared to normal.

In the list of upregulated miRNAs as determined from our profiling study, miR-141 was found to have the highest expression in metastatic SOC compared to normal, with log2-fold changes more than 9 (Table 2). MiR-141 is a member of the miR-200 family, alongside other members, namely $\mathrm{miR}-200 \mathrm{a} / \mathrm{b} / \mathrm{c}$ and $\mathrm{miR}-429$ [24]. In this paper, $\mathrm{miR}-200 \mathrm{a} / \mathrm{b} / \mathrm{c}$ were also shown to have high expression in metastatic SOC compared to normal, with log2-fold changes more than 7 . The high expression of the miR-200 family in metastatic SOC compared to normal was also observed in previous profiling studies $[10,19,22]$. The high expression of the miR-200 family found in our study has led us to choose miR-141 and miR-200a to be further experimented (in vitro) to determine their interactions with the targeted genes and their roles in ovarian cancer growth and progression.

The bioinformatics analysis conducted in this study has shown that DLC-1 and ZEB2 were the predicted targeted genes for miR-141 and miR-200a, respectively, as shown in Figure 3. The gene $D L C-1$ is a tumor suppressor, and ZEB2 is a transcriptional repressor, which have been shown to be downregulated in ovarian cancers compared to normal tissues [25]. We hypothesized that miR-141 and miR-200a inhibition in ovarian cancer cells will increase the expression levels of their targeted genes. Our results demonstrated increased DLC-1 and ZEB2 expression following miR-141 and miR-200a inhibition, respectively (Figure 4). However, the changes in fold change observed were not statistically significant, and some may argue that the changes seemed minimal to exert substantial effects on cancer cell regulation (1.2- to 1.5-fold changes). We recognized these limitations; nevertheless, the findings in this paper may provide preliminary insights on the interaction between miR-141 and miR-200a and DLC-1 and ZEB2, respectively, in SOC. A study by Bendroaite et al. confirmed that ZEB2 is the targeted gene for the miR-200 family in ovarian cancer using the luciferase-ZEB2 $3^{\prime}$-UTR reporter 
constructs and miR-200 family expression plasmids [25]. The regulation of DLC-1 by miR-141 has also been shown in colorectal cancer [26]. Our study is the first to demonstrate the possible regulation of DLC1 by miR-141 in SOC; thus, experiments using the luciferase reporter assay can be performed in the future to confirm DLC-1 as the targeted gene for miR-141. DLC- 1 acts as a tumor-suppressor gene, and its low expression was associated with poor prognosis in lung cancer [27]. A meta-analysis study involving 1815 cancer cases demonstrating a low expression of DLC-1 was associated with advanced stages of cancer, including ovarian cancer [28].

The overexpression of miR-141 in ovarian cancer cell lines was shown to cause increased resistance to cisplatin, thus enhancing cancer growth [29]. MiR-141 and miR-200a were also found to have effects on ovarian tumor growth through the oxidative stress response control mechanism. Increased expression of these miRNAs has been shown to increase tumor growth in mouse models [11]. We have shown that cell viability was significantly reduced in ovarian cancer cells following transfection with antimiR-141, as shown in Figure 5a. In view of our findings, we proposed that treatment with antimiR-141 may result in an antitumorigenic effect via a reduction in cell viability. However, significant reduction in cell viability was only seen in cells treated with anti-miR-141 but not with anti-miR-200a. This may be due to the different molecular pathways or cell signals that promote ovarian cancer growth in relation to these miRNAs, such as the P13K pathway and the estrogen receptor pathway [30].

In this study, we observed that transfection with miRNA inhibitors led to a significant rise in ovarian cancer cell migration and invasion, as shown in Figure $5 c$,d and Figure $5 e, f$. Cancer progression was largely attributed to the epithelial-to-mesenchymal transition (EMT), which was associated with tumor migration, invasion, metastasis and treatment resistance [31]. EMT is characterized by the loss of E-cadherin, an important adhesion molecule of epithelial cells, resulting from the overexpression of transcriptional repressors ZEB1 or ZEB2, Snail 1/2 and TWIST [32,33]. Suppression of E-cadherin expression leads to the acquisition of mesenchymal and migratory characteristics and metastasis [34]. In ovarian cancer, members of the miR-200 family have been implicated with EMT in which ZEB1 and ZEB2 were their targets [25]. Upregulation of the miR-200 family is thought to impede EMT and suppress metastasis by augmenting E-cadherin expression through the direct targeting of ZEB1 and ZEB2. Overexpression of the miR-200 family was also found to inhibit cell mobility significantly in ovarian cancer cells [12]. Conversely, the miR-200 family repression leads to reduced E-cadherin expression and loss of cell-cell adhesion, thus enhancing cell invasion and migration [35]. This understanding is further substantiated by the results of our cell migration and invasion experiments.

Nevertheless, it is notable that treatment with miRNA inhibitors led to enhanced cell migration in Caov3 cells, but migration was significantly reduced by almost 30\% in SKOV3 cells. This discrepancy may be attributed to the sources of the cell lines used. The cell line Caov3 was derived from primary epithelial ovarian adenocarcinoma, while SKOV3 was established from the ascites of epithelial ovarian adenocarcinoma (American Type Culture Collection, Manassas, VA, USA). Moreover, the putative histological subtypes of the two cell lines used may also contribute to the discrepancy. Both Caov3 and SKOV3 cell lines have TP53 mutations, but SKOV3 cells also have ARID1A mutation, making Caov3 putative histology as serous, while SKOV3 very much represents the endometrioid histological subtype [36]. As such, cell lines with molecular properties which best represent the histological subtype under study would be an important aspect to be addressed in future studies.

Although cell migration was seen to increase only in Caov3 cells, cell invasion was observed to rise significantly in both Caov3 and SKOV3 cells following transfection with miRNA inhibitors, as shown in Figure 5e,f. This may be due to the differences in the molecular process of cell migration and invasion, in which cancer cells can only invade if migration occurs but migration may occur without invasion [37]. Migration refers to the movement of cancer cells from one site to another site, but invasion involves the rupture of the cell membrane with the aid of the protein matrix metalloproteases [37]. 


\section{Conclusions}

In conclusion, our study has demonstrated the differentially expressed miRNAs in metastatic SOC compared to normal, with miR-19a identified as a novel miRNA to be upregulated. Possible regulation of $D L C-1$ by miR-141 in SOC was shown, and this finding may serve as an impetus for future experiments to be done more extensively. The functional roles of miR-141 and miR-200a add to the existing knowledge of how these miRNAs contribute to SOC carcinogenesis. This report contributes to the understanding of miRNA expression profiling in metastatic SOC and the molecular aspects underlying its growth and progression.

Author Contributions: Conceptualization, N.M.M. and R.A.R.A.; methodology, validation, formal analysis and investigation, Z.O.; writing-original draft preparation, N.M.M. and Z.O.; writing-review and editing, N.M.M., R.A.R.A., N.A.W., N.W.M.N., M.H.M., S.F.I. and A.A.H.; supervision, N.M.M. and R.A.R.A.; project administration, N.M.M.; funding acquisition, N.M.M. All authors have read and agreed to the published version of the manuscript.

Funding: This research received funding from the Fundamental Research Grant, Ministry of Education, Malaysia (FRGS/1/2014/SKK01/UKM/02/2).

Acknowledgments: We thank the staff and members of the Department of Physiology, Faculty of Medicine and Universiti Kebangsaan Malaysia Medical Centre (UKMMC) for their support and help in completing this research project. A special thanks to Ms. Norermi Firzana Ahmad Alfian for helping in the preparation of the graphic illustrations.

Conflicts of Interest: The authors declare no conflicts of interest.

\section{Appendix A}

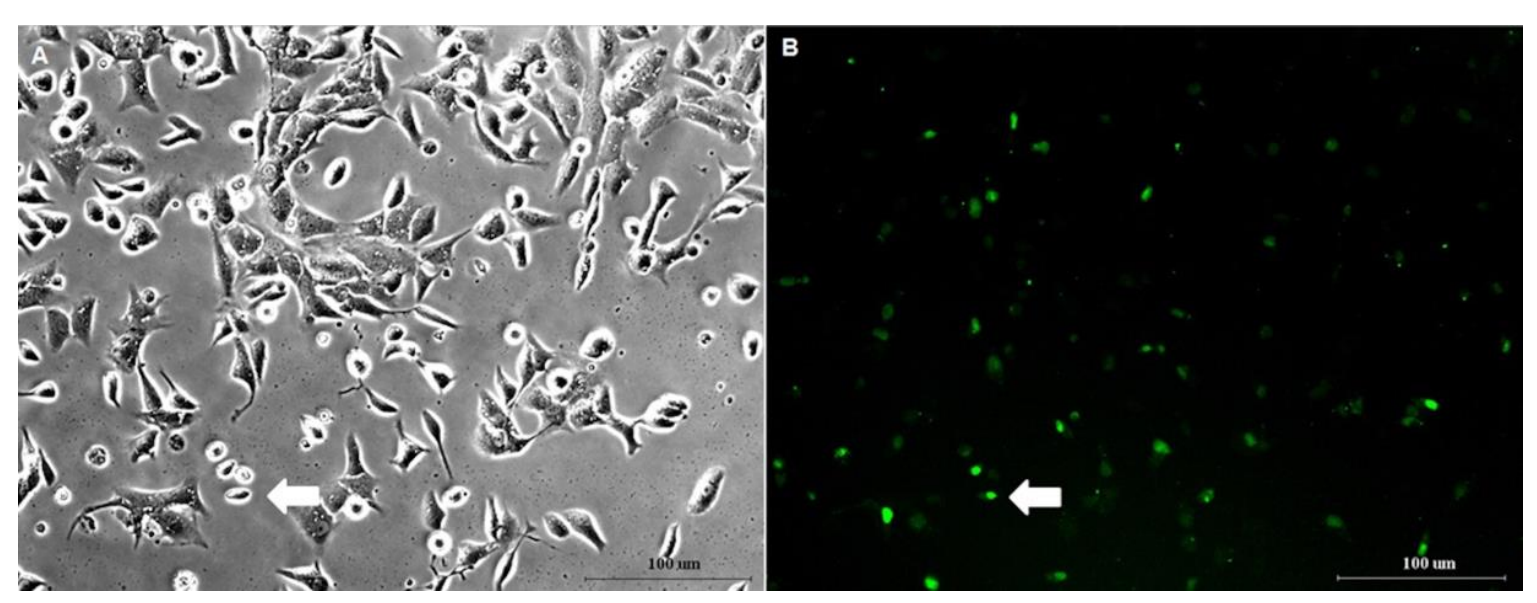

Figure A1. (A) SKOV-3 cells under fluorescence microscope (phase contrast), x200. (B) SKOV-3 cells under fluorescence microscope (FITC), x200. Green fluorecein (arrow) indicates miR-141 inhibitor successfully transfected into the cell. Transfection efficiency was between $60-70 \%$.

\section{References}

1. Reid, B.M.; Permuth, J.B.; Sellers, T.A. Epidemiology of ovarian cancer: A review. Cancer Biol. Med. 2017, 14, 9-32.

2. Bell, D.A. Origins and molecular pathology of ovarian cancer. Mod. Pathol. 2005, 18, S19-S32. [CrossRef]

3. Salazar, C.; Campbell, I.G.; Gorringe, K.L. When is "type I" ovarian cancer not "type I"? indications of an out-dated dichotomy. Front. Oncol. 2018, 8, 654. [CrossRef] [PubMed]

4. Bowtell, D.D.; Bohm, S.; Ahmed, A.A.; Aspuria, P.J.; Bast, R.C., Jr.; Beral, V.; Berek, J.S.; Birrer, M.J.; Blagden, S.; Bookman, M.A.; et al. Rethinking ovarian cancer Ii: Reducing mortality from high-grade serous ovarian cancer. Nat. Rev. Cancer 2015, 15, 668-679. [CrossRef] [PubMed]

5. Chen, V.W.; Ruiz, B.; Killeen, J.L.; Cote, T.R.; Wu, X.C.; Correa, C.N. Pathology and classification of ovarian tumors. Cancer 2003, 97, 2631-2642. [CrossRef] [PubMed] 
6. Agostini, M.; Ganini, C.; Candi, E.; Melino, G. The role of noncoding rnas in epithelial cancer. Cell Death Discov. 2020, 6, 13. [CrossRef] [PubMed]

7. Ghafouri-Fard, S.; Shoorei, H.; Taheri, M. Mirna profile in ovarian cancer. Exp. Mol. Pathol. 2020, 113, 104381. [CrossRef]

8. Shah, M.Y.; Ferrajoli, A.; Sood, A.K.; Lopez-Berestein, G.; Calin, G.A. Microrna therapeutics in cancer-An emerging concept. EBioMedicine 2016, 12, 34-42. [CrossRef] [PubMed]

9. Niu, K.; Shen, W.; Zhang, Y.; Zhao, Y.; Lu, Y. Mir-205 promotes motility of ovarian cancer cells via targeting ZEB1. Gene 2015, 574, 330-336. [CrossRef] [PubMed]

10. Ibrahim, F.F.; Jamal, R.; Syafruddin, S.E.; Ab Mutalib, N.S.; Saidin, S.; MdZin, R.R.; Hossain Mollah, M.M.; Mokhtar, N.M. Microrna-200c and Microrna-31 regulate proliferation, colony formation, migration and invasion in serous ovarian cancer. J. Ovarian Res. 2015, 8, 56. [CrossRef] [PubMed]

11. Mateescu, B.; Batista, L.; Cardon, M.; Gruosso, T.; de Feraudy, Y.; Mariani, O.; Nicolas, A.; Meyniel, J.P.; Cottu, P.; Sastre-Garau, X.; et al. Mir-141 and Mir-200a act on ovarian tumorigenesis by controlling oxidative stress response. Nat. Med. 2011, 17, 1627-1635. [CrossRef] [PubMed]

12. Hu, X.D.; Macdonald, M.; Huettner, P.C.; Feng, Z.; El Naqa, I.M.; Schwarz, J.K.; Mutch, D.G.; Grigsby, P.W.; Powell, S.N.; Wang, X. A Mir-200 microrna cluster as prognostic marker in advanced ovarian cancer. Gynecol. Oncol. 2009, 114, 457-464. [CrossRef] [PubMed]

13. Yanaihara, N.; Noguchi, Y.; Saito, M.; Takenaka, M.; Takakura, S.; Yamada, K.; Okamoto, A. Microrna gene expression signature driven by Mir-9 overexpression in ovarian clear cell carcinoma. PLoS ONE 2016, 11, e0162584. [CrossRef] [PubMed]

14. Prahm, K.P.; Hogdall, C.; Karlsen, M.A.; Christensen, I.J.; Novotny, G.W.; Hogdall, E. Identification and validation of potential prognostic and predictive mirnas of epithelial ovarian cancer. PLOS ONE 2018, 13, e0207319. [CrossRef]

15. Wan, Y.W.; Mach, C.M.; Allen, G.I.; Anderson, M.L.; Liu, Z. On the reproducibility of tcga ovarian cancer microrna profiles. PLoS ONE 2014, 9, e87782. [CrossRef]

16. Bai, H.; Cao, D.; Yang, J.; Li, M.; Zhang, Z.; Shen, K. Genetic and epigenetic heterogeneity of epithelial ovarian cancer and the clinical implications for molecular targeted therapy. J. Cell Mol. Med. 2016, 20, 581-593. [CrossRef]

17. Vang, S.; Wu, H.T.; Fischer, A.; Miller, D.H.; MacLaughlan, S.; Douglass, E.; Comisar, L.; Steinhoff, M.; Collins, C.; Smith, P.J.; et al. Identification of ovarian cancer metastatic mirnas. PLoS ONE 2013, 8, e58226. [CrossRef]

18. Meng, X.; Muller, V.; Milde-Langosch, K.; Trillsch, F.; Pantel, K.; Schwarzenbach, H. Diagnostic and prognostic relevance of circulating exosomal Mir-373, Mir-200a, Mir-200b and Mir-200c in patients with epithelial ovarian cancer. Oncotarget 2016, 7, 16923-16935. [CrossRef]

19. Chao, A.; Lin, C.Y.; Lee, Y.S.; Tsai, C.L.; Wei, P.C.; Hsueh, S.; Wu, T.I.; Tsai, C.N.; Wang, C.J.; Chao, A.S.; et al. Regulation of ovarian cancer progression by Microrna-187 through targeting disabled Homolog-2. Oncogene 2012, 31, 764-775. [CrossRef]

20. Creighton, C.J.; Fountain, M.D.; Yu, Z.; Nagaraja, A.K.; Zhu, H.; Khan, M.; Olokpa, E.; Zariff, A.; Gunaratne, P.H.; Matzuk, M.M.; et al. Molecular profiling uncovers a P53-associated role for Microrna-31 in inhibiting the proliferation of serous ovarian carcinomas and other cancers. Cancer Res. 2010, 70, 1906-1915. [CrossRef]

21. Gong, G.; Lin, T.; Yuan, Y. Integrated analysis of gene expression and DNA methylation profiles in ovarian cancer. J. Ovarian Res. 2020, 13, 30. [CrossRef] [PubMed]

22. Wyman, S.K.; Parkin, R.K.; Mitchell, P.S.; Fritz, B.R.; O’Briant, K.; Godwin, A.K.; Urban, N.; Drescher, C.W.; Knudsen, B.S.; Tewari, M. Repertoire of micrornas in epithelial ovarian cancer as determined by next generation sequencing of small rna cdna libraries. PLoS ONE 2009, 4, e5311. [CrossRef] [PubMed]

23. Dahiya, N.; Sherman-Baust, C.A.; Davidson, T.L.; Wang, B.; Shih Ie, M.; Zhang, Y.; Wood, W., 3rd; Becker, K.G.; Morin, P.J. Microrna expression and identification of putative mirna targets in ovarian cancer. PLoS ONE 2008, 3, e2436. [CrossRef] [PubMed]

24. Vaksman, O.; Stavnes, H.T.; Kaern, J.; Trope, C.G.; Davidson, B.; Reich, R. Mirna profiling along tumour progression in ovarian carcinoma. J. Cell Mol. Med. 2011, 15, 1593-1602. [CrossRef] [PubMed] 
25. Bendoraite, A.; Knouf, E.C.; Garg, K.S.; Parkin, R.K.; Kroh, E.M.; O’Briant, K.C.; Ventura, A.P.; Godwin, A.K.; Karlan, B.Y.; Drescher, C.W.; et al. Regulation of Mir-200 family micrornas and zeb transcription factors in ovarian cancer: Evidence supporting a mesothelial-to-epithelial transition. Gynecol. Oncol. 2010, 116, 117-125. [CrossRef]

26. Wu, P.P.; Zhu, H.Y.; Sun, X.F.; Chen, L.X.; Zhou, Q.; Chen, J. Microrna-141 regulates the tumour suppressor Dlc1 in colorectal cancer. Neoplasma 2015, 62, 705-712. [CrossRef]

27. Wang, D.; Qian, X.; Rajaram, M.; Durkin, M.E.; Lowy, D.R. Dlc1 is the principal biologically-relevant down-regulated Dlc family member in several cancers. Oncotarget 2016, 7, 45144-45157. [CrossRef]

28. Jiang, Y.; Li, J.M.; Luo, H.Q. Clinicopathological significance of Dlc-1 expression in cancer: A meta-analysis. Asian Pac. J. Cancer Prev. 2015, 16, 7255-7260. [CrossRef]

29. Van Jaarsveld, M.T.; Helleman, J.; Boersma, A.W.; van Kuijk, P.F.; van Ijcken, W.F.; Despierre, E.; Vergote, I.; Mathijssen, R.H.; Berns, E.M.; Verweij, J.; et al. Mir-141 regulates Keap1 and modulates cisplatin sensitivity in ovarian cancer cells. Oncogene 2013, 32, 4284-4293. [CrossRef]

30. Gewinner, C.; Wang, Z.C.; Richardson, A.; Teruya-Feldstein, J.; Etemadmoghadam, D.; Bowtell, D.; Barretina, J.; Lin, W.M.; Rameh, L.; Salmena, L.; et al. Evidence that inositol polyphosphate 4-phosphatase type ii is a tumor suppressor that inhibits Pi3k signaling. Cancer Cell 2009, 16, 115-125. [CrossRef]

31. Thiery, J.P.; Acloque, H.; Huang, R.Y.; Nieto, M.A. Epithelial-mesenchymal transitions in development and disease. Cell 2009, 139, 871-890. [CrossRef]

32. Kang, Y.; Massague, J. Epithelial-mesenchymal transitions: Twist in development and metastasis. Cell 2004, 118, 277-279. [CrossRef]

33. Vandewalle, C.; Comijn, J.; De Craene, B.; Vermassen, P.; Bruyneel, E.; Andersen, H.; Tulchinsky, E.; Van Roy, F.; Berx, G. Sip1/Zeb2 induces emt by repressing genes of different epithelial cell-cell junctions. Nucleic Acids Res. 2005, 33, 6566-6578. [CrossRef] [PubMed]

34. Prieto-Garcia, E.; Diaz-Garcia, C.V.; Garcia-Ruiz, I.; Agullo-Ortuno, M.T. Epithelial-to-mesenchymal transition in tumor progression. Med. Oncol. 2017, 34, 122. [CrossRef] [PubMed]

35. Korpal, M.; Lee, E.S.; Hu, G.; Kang, Y. The Mir-200 family inhibits epithelial-mesenchymal transition and cancer cell migration by direct targeting of e-cadherin transcriptional repressors Zeb1 and Zeb2. J. Biol. Chem. 2008, 283, 14910-14914. [CrossRef] [PubMed]

36. Beaufort, C.M.; Helmijr, J.C.; Piskorz, A.M.; Hoogstraat, M.; Ruigrok-Ritstier, K.; Besselink, N.; Murtaza, M.; van Ijcken, W.F.; Heine, A.A.; Smid, M.; et al. Ovarian cancer cell line panel (Occp): Clinical importance of in vitro morphological subtypes. PLoS ONE 2014, 9, e103988. [CrossRef] [PubMed]

37. Trepat, X.; Chen, Z.; Jacobson, K. Cell migration. Compr. Physiol. 2012, 2, 2369-2392. [PubMed] 